\title{
Confirmation test for hysteroscopic sterilization: a descriptive study of patient tolerability and impressions
}

This article was published in the following Dove Press journal:

Patient Preference and Adherence

3 April 2013

Number of times this article has been viewed

\section{Hector O Chapa \\ Gonzalo Venegas}

Department of Obstetrics and Gynecology, Methodist Medical and Women's Specialty Center, Dallas, TX, USA
Correspondence: Hector O Chapa Women's Specialty Center, Dallas, Chapa Medical Consulting, 2042 Marydale Rd, Dallas, TX 75208, USA

$\mathrm{Tel}+\mathrm{I} 2143348116$

Email chapamd@icloud.com
Background: This retrospective descriptive study describes patient follow-up and tolerability of the post-hysteroscopic sterilization confirmation test.

Methods: Recruitment for the original sterilization procedure was from January 2008 to March 2009; subsequent confirmation test (hysterosalpingogram) capture was from March 2008 to July 2009. Patients were given a $10 \mathrm{~cm}$ visual analog pain scale during the hysteroscopic sterilization procedure, and took the scale with them as a take-home sheet. Following hysterosalpingography (HSG), patients received a follow-up phone call within 24 hours, and were asked to rate their pain during the hysterosalpingogram as well as during the first 2 hours following the test.

Results: Eighty-nine hysteroscopic sterilizations were performed under local paracervical block and oral nonsteroidal medication. The median immediate post-sterilization visual analog pain score was 1.9 (range 1.7-2.1, 95\% confidence interval [CI] 1.3-1.5). Of the 89 sterilization procedures, $79 \%(\mathrm{n}=70)$ patients underwent a confirmation test using HSG. Ten percent $(n=7)$ of the hysterosalpinograms were performed at least 3 months after sterilization (mean 17 [range 14-20] weeks). Median intratest visual analog pain score overall $(\mathrm{n}=70)$ was 1.8 (range 1.6-1.9, 95\% CI 1.5-1.9). Following the test, the median visual analog pain score was 1.7 (range $1.6-1.9,95 \%$ CI $1.4-0.18$ ). Of the 70 patients who participated in visual analog pain score capture, 64 had a paper copy of the scale had six had it via email. Of the 19 who did not complete hysterosalpinography, five were lost to follow-up. Reasons given by the remaining 14 for noncompliance with hysterosalpinography were: a busy schedule/childcare issues (62\%), fear of the test (13\%), trust in the sterilization procedure alone (13\%), and forgetting the appointment (12\%). Of the $70 \mathrm{HSGs}$ performed, 69 revealed satisfactory micro insert positions with bilateral occlusion; one was unilaterally patent at 13 weeks post-sterilization, with satisfactory micro insert position. Repeat testing 10 weeks later documented bilateral occlusion.

Conclusion: Confirmation testing for hysteroscopic sterilization is well tolerated, with favorable patient impressions after completion.

Keywords: Female permanent birth control, female sterilization, Essure confirmation test, contraception

\section{Introduction}

On March 23, 2010, the Patient Protection and Affordable Care Act was signed into US law, and the constitutionality of the law passage was upheld on June 28, 2012, by the US Supreme Court. One of the provisions within this act calls for women's access to contraceptive options to be free of patient costs, under the coverage of private insurance. In the US, female sterilization is the second most commonly used form of contraception overall, and is the most frequently used method among married women 
and those over 30 years of age. ${ }^{1}$ Minimally invasive options for female sterilization have allowed the procedure to be performed under light to no anesthesia as well as within an office setting if desired. ${ }^{2}$

Numerous studies have been published on patient satisfaction and acceptability during and after hysteroscopic sterilization. ${ }^{3-6}$ Other researchers have published their experience and proposed analgesia protocols during hysterosalpinography for infertility. ${ }^{7-9}$ Traditionally, hysterosalpinography for infertility is done under high dye pressure instillation in an attempt to overcome proximal ostial spasm and/or mucus plugs, so hysterosalpinography (HSG) for infertility may be accompanied by pain. ${ }^{8}$ In a randomized prospective study, Zhu et al evaluated the effect of warm (37 degrees) versus cold contrast media during hysterosalpinography for infertility in an attempt to reduce reflex tubal/uterine spasm. Patients' visual analog scale (VAS) pain scores during hysterosalpinography were significantly lower in the warm medium group initially, but showed no statistically significant difference 30 minutes following injection. Temperature of the medium showed a linear association with VAS pain score. The total number of vasovagal episodes was higher in the cold medium group. ${ }^{8}$

Chauhan et al similarly attempted to reduce the pain associated with hysterosalpinography for infertility by using an intracervical anesthetic block. ${ }^{9}$ Their prospective randomized study divided patients using a computer-generated table into two groups of 50 women each. In the study group, women received an intracervical block along with their premedication, whereas the controls received premedication alone. According to the authors, "each patient was asked to rate her pain at six different points of time (T1-T6) during hysterosalpinography using a VAS and verbal descriptive score". Reduction of pain from placement of a tenaculum was observed until the end of procedure (T3-T6) with intracervical block $(P<0.05)$. Pain remained at a statistically lower level during the most painful steps, ie, traction of the cervix and insertion of dye $(P=0.001)$ in the study group as compared with the control group. ${ }^{9}$ Information gathered from such reports may be helpful outside of hysterosalpinography for infertility. For example, our group's protocol for hysteroscopic sterilization performed in the office includes instillation of slightly warmed saline into the cavity as well as paracervical block analgesia (1\% mepivacaine $30 \mathrm{~mL}$ diluted in $20 \mathrm{~mL}$ of normal saline). Our in-office paracervical block is administered at the cervicovaginal reflection via a 22-gauge syringe at the 4, 8, 2, and 10 o'clock positions. All patients receive one tablet of naproxen sodium $550 \mathrm{mg}$ approximately 30 minutes before sterilization. No anxiolytic or intravenous medication is administered.
However, reports such as ours and others have focused on infertility-based testing, not post-sterilization. A search of the Medline, PubMed, and Medscape databases did not retrieve any published articles on patient tolerability or impressions of the post-hysteroscopic sterilization confirmation test currently required by the US Food and Drug Administration. Therefore, we sought to be the first to investigate patient tolerability and impressions during and after this radiographic intervention. This study was not intended, powered, or designed to evaluate the "best" medication regimen prior to hysterosalpinography.

This is a subanalysis of our previously published research on patient preference and attitudes towards options for permanent birth control. ${ }^{10}$ In the original publication, research staff at the Women's Specialty Center in Dallas investigated patient preferences for traditional laparoscopic tubal occlusion versus a hysteroscopic approach pre procedure. As stated in our recent article, "a total of 100 patients desiring permanent contraception were offered the two procedures. A description of each procedure was developed and read to each patient by a research nurse on site. Patients were then asked to respond to a questionnaire concerning options. Final informed consent, procedure review, and procedural date determination were provided by a physician upon completion of the questionnaire. Patients were not allowed to change their questionnaire responses after completion. No interviewer or physician input was allowed during the questionnaire. The study was completed in English or Spanish, as per patient request, by a bilingual/fluent speaker. Physicians completing informed consent were unaware of the questionnaire responses." $" 10$

Of the 100 patients recruited, 94 opted for hysteroscopic intervention after a detailed description of both surgical interventions. Of these, 89 (95\%) who opted for hysteroscopic sterilization proceeded to and completed sterilization. Of the six women who elected laparoscopic sterilization, 4 (67\%) actually proceeded to surgery. The remainder (five in the hysteroscopic group, two in the laparoscopic group) cancelled because of lack of financial resources (copay funds, ie, those unable to provide their required financial portion for the procedure). ${ }^{10}$

The original analysis did not include data on subsequent completion of post-sterilization hysterosalpinography or tolerance of the test. Therefore, the objective of the current study was to describe patient tolerability of the post-hysteroscopic sterilization confirmation test when performed under nonsteroidal analgesia and low pressure. This was not a randomized study of the effectiveness of any premedication regimen; rather, we sought to be fill the void in the literature regarding 
pain associated with that test. As in similar published reports, we chose to use a previously published vertical VAS to measure pain (Figure 1). ${ }^{11}$

The VAS is a continuous scale comprised of a horizontal or vertical line, usually $10 \mathrm{~cm}(100 \mathrm{~mm})$ in length, anchored by two verbal descriptors, one for each symptom extreme. ${ }^{11}$ The scale is most commonly anchored by "no pain" (score 0 ) and "pain as bad as it could be" or "worst imaginable pain" (score 10). The recall period for reporting varies based on the clinical setting, but most commonly respondents are asked to report "current" pain intensity or pain intensity "in the last 24 hours". ${ }^{11}$ The ethical review board at the Women's Specialty Center granted approval for both the original and current investigation. Patients were not financially incentivized to participate in the study, nor were these studies funded by industry.

\section{Materials and methods}

The original sterilization procedure recruitment and study was conducted from January 2008 to March 2009; subsequent confirmation test capture was from March 2008 to July 2009. Patients received and answered a $10 \mathrm{~cm}$ VAS for pain during their hysteroscopic sterilization, and took the scale with them as a take-home sheet (Figure 1). Patients were instructed on interpretation of the VAS as " 0 " being absent pain and "10" representing maximum pain. Following hysterosalpinography, patients received a follow-up phone call within 24 hours, and were asked to rate their pain during the procedure as well as during the first 2 hours after the test.

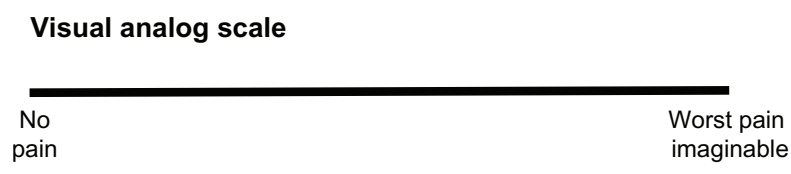

\section{Verbal descriptor scale}

\begin{tabular}{cccccc}
\hline No & Mild & Moderate & Severe & Very & Worst \\
pain & pain & pain & pain & severe & pain \\
& & & & pain & imaginable
\end{tabular}

Numeric rating scale

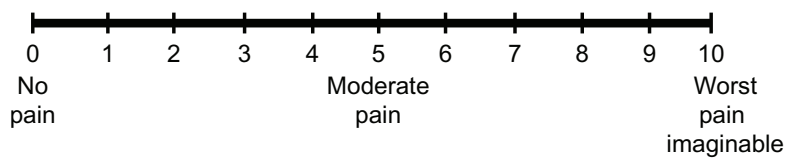

Figure I Patient visual analog pain scale.

Adapted from Hamill-Ruth RJ, Marohn ML. Evaluation of pain in the critically ill patient. Crit Care Clin. 1999;15(I):35-54 (used with permission, Elsevier License 3060890426622).
To confirm that participants had the VAS scale for their follow-up, they were sent another copy one week prior to their anticipated appointment for hysterosalpinography. This was done by our research associate via standard mail. At time of the follow-up call, patients were asked to confirm their possession of the scale during questioning. Those without a copy were sent an immediate email with the VAS attached. VAS capture was performed by an independent research assistant not associated with authorship of this manuscript. Although VAS scores were obtained during the actual initial hysteroscopic sterilizations (under local anesthesia), the focus of this study was the post-procedure confirmation test.

As per professional agreement, post-hysteroscopic sterilization confirmation tests are performed at an inner city Dallas radiology clinic staffed by nonresident physicians. Patients are instructed to self-medicate with oral ibuprofen $600 \mathrm{mg} 1-2$ hours before their scheduled appointment. Neither intravenous nor oral sedation is administered during the fluoroscopic examination. Per protocol, the intrauterine catheter is placed via a vaginal speculum. A scout image is obtained, and 4-6 anterior-posterior uterine images are captured after dye instillation. The protocol states low-pressure fill, with less than $5 \mathrm{~mL}$ of dye recommended for uterine fill or more as needed at the physician's discretion. Hysterosalpinographies were interpreted by the performing radiologists.

\section{Results}

The sociodemographics of our study population is shown in Table 1. Eighty-nine hysteroscopic sterilizations were performed under local paracervical block and oral nonsteroidal medication. The median immediate poststerilization VAS was 1.9 (range 1.7-2.1, 95\% confidence interval [CI] 1.3-1.5). Of the 89 women who completed hysteroscopic sterilization, 79\% $(\mathrm{n}=70)$ underwent confirmation testing. Ten percent $(n=7)$ completed the test more than 3 months after sterilization (mean 17 [range 14-20] weeks). All 70 patients reported self-medicating with nonsteroidal anti-inflammatory agents within 2 hours before performance of hysterosalpinography. Of the 70 patients who participated in VAS capture, 64 had a paper copy of the scale and six had it via email. Median intratest VAS score overall $(\mathrm{n}=70)$ was 1.8 (range $1.6-1.9,95 \%$ CI $1.5-1.9)$. Following the test, the median VAS was 1.7 (range 1.6-1.9, 95\% CI 1.4-1.8). Of the 19 who did not complete their HSG, five were lost to follow-up. Reasons given by the remaining 14 for noncompliance with hysterosalpinography were: busy schedule/childcare issues (62\%), fear of the test (13\%), trust in the sterilization procedure alone (13\%), and $12 \%$ forgetting the appointment. 
Table I Patient demographics per group: original recruited sample, and groups compliant and noncompliant with hysterosalpingography*

\begin{tabular}{|c|c|c|c|}
\hline & \multicolumn{3}{|l|}{ Study cohort } \\
\hline & $\begin{array}{l}\text { Original } \\
(n=100)\end{array}$ & $\begin{array}{l}\text { HSG compliant } \\
(n=70)\end{array}$ & $\begin{array}{l}\text { HSG noncompliant } \\
(\mathrm{n}=19)\end{array}$ \\
\hline \multicolumn{4}{|l|}{ Ethnicity } \\
\hline AA & $(34 \%)$ & $29(42 \%)$ & $6(32 \%)$ \\
\hline Caucasian & $(29 \%)$ & 15 (22\%) & $4(21 \%)$ \\
\hline Hispanic & $(37 \%)$ & $26(36 \%)$ & $9(47 \%)$ \\
\hline \multicolumn{4}{|l|}{ Age (years) } \\
\hline & $34-48($ mean $=44)$ & $38-48($ mean $=45)$ & $34-39($ mean $=37)$ \\
\hline \multicolumn{4}{|l|}{ Living children } \\
\hline$\leq 3$ & $(64 \%)$ & 57 (82\%) & $8(42 \%)$ \\
\hline $4-5$ & $(32 \%)$ & $13(18 \%)$ & $10(53 \%)$ \\
\hline$\geq 6$ & $(4 \%)$ & 0 & $\mathrm{I}(5 \%)$ \\
\hline \multicolumn{4}{|l|}{ Employed } \\
\hline & $(93 \%)$ & 66 (94\%) & I5 (79\%) \\
\hline \multicolumn{4}{|l|}{ Past surgery } \\
\hline & $(16 \%)$ & $15(21 \%)$ & 0 \\
\hline \multicolumn{4}{|c|}{ Highest grade completed } \\
\hline$<10$ & $(2 \%)$ & 0 & 0 \\
\hline $10-12$ & $(63 \%)$ & 59 (84\%) & $6(32 \%)$ \\
\hline College 2 yr & $(14 \%)$ & $10(14 \%)$ & $13(68 \%)$ \\
\hline College $>2 \mathrm{yr}$ & $(12 \%)$ & $\mathrm{I}(2 \%)$ & 0 \\
\hline
\end{tabular}

Note: *One hundred patients were initially recruited into the study, 89 proceeded to hysteroscopic sterilization, of which 70 completed follow-up following hysterosalpingography.

Abbreviations: AA, African-American; HSG, hysterosalpingography.

Of the 70 HSGs performed, 89 showed satisfactory micro insert positions with bilateral occlusion; one was unilaterally patent with satisfactory microinsert positions bilaterally. Repeat testing 10 weeks later documented bilateral occlusion.

\section{Discussion}

Advances in minimally invasive technologies have allowed surgical interventions traditionally performed in the operating room setting and under general anesthesia to be accomplished in a more patient-friendly in-office setting and atmosphere. The first hysteroscopic sterilization device (Essure ${ }^{\circledR}$, Conceptus Inc, Mountain View, CA, USA) was approved by the US Food and Drug Administration (FDA) in 2002. Since then, numerous analgesia protocols and outcomes have validated the effectiveness and tolerability of the Essure device. ${ }^{12-16}$ However, no published data exist on the tolerability or patient impressions of the post-procedure hysterosalpingraphy confirmation test required by the FDA. In this study, we documented low VAS pain scores.

Seventy-nine percent of our sterilization subjects complied with hysterosalpinography testing, while 19 patients had not undergone confirmation testing by 6 months following sterilization.

Although the noncompliance group was very small, our findings are similar to those of Howard et al, who reported that younger patients with higher parity (more than three children) showed lower compliance with follow-up. Our findings concur with theirs. These authors also noted that women undergoing the Essure procedure at the campus with a dedicated protocol to ensure compliance had an almost fourfold higher odds of compliance with hysterosalpinography. ${ }^{17}$ Our institution uses a dedicated nurse to send monthly reminders to our hysteroscopic sterilization patients, possibly accounting for the relatively high compliance rate of $79 \%$. As of December 2012, no pregnancies have occurred in the women who underwent the original 89 sterilization procedures.

According to the manufacturer, the post Essure confirmation test differs from a standard infertility hysterosalpingogram in that a slow-pressure fill is advised, along with a low-volume uterine fill. ${ }^{18}$ To our knowledge, we are the first group to report on this subject. In that lies the greatest scientific strength of our investigation. In addition, use of a standardized VAS scale which the patients had in their possession brings objectivity to an otherwise subjective issue, ie, pain. We attempted to eliminate observer bias by having an independent research assistant collect the VAS scores and by conducting interviews based on a prewritten script.

We chose to contact the patients at 24 hours post procedure instead of immediately following the procedure for ease of capture/logistics. Because we were not the physicians (radiologists) performing the hysterosalpinograms, VAS 
scores were not obtained during the procedure, nor were the radiologists performing the HSGs aware of our study. It was felt that knowledge of the VAS reporting could influence performance of hysterosalpinography by the radiologists. Patients were instructed by us (authors) not to disclose the study to the radiology staff. Similar to our protocol, others have published similar assessments of pain hours after the index procedure. Updike et al studied patients undergoing abdominal surgical procedures via a planned vertical midline incision under general anesthesia. Patients were assigned randomly to receive either ropivacaine $0.5 \%$ or normal saline solution injected in the subcuticular tissue and fascia before the incision of each. All patients received morphine after the operation, using a patientcontrolled analgesia device. Morphine consumption was measured during the postoperative period at intervals of 0-6 hours, 6-12 hours, 12-24 hours, and 24-48 hours. Postoperative pain was assessed using VAS at 6, 12, 24, and 48 hours after conclusion of the procedure. They concluded that pre-emptive analgesia with ropivacaine $0.5 \%$ given before skin incision did not decrease postoperative analgesic use in their patients. ${ }^{19}$

Similarly, Tripp et al reported VAS scores collected 24 hours postoperatively from adolescents and adults following anterior cruciate ligament surgery. They concluded that adolescents reported greater pain, catastrophizing, and anxiety than adults at 24 hours. ${ }^{20}$ We are aware that the reliability of pain score recollection obtained at 24 hours post procedure may be different than that collected immediately postoperatively. In additional, we acknowledge that data capture may have been different if performed 2 hours after hysterosalpinography rather than after 24 hours. However, for logistic reasons, we opted for our present model. This is one of the limitations of our design.

Despite the novelty of our study, we do acknowledge its limitations. We elected to have patients report their pain only until 2 hours after completion. We hypothesized that most uterine spasm/cramping would be limited to these first two hours and therefore did not collect data after that period. We were unable to locate any published data in PubMed or Medline describing time for persistence of discomfort following hysterosalpinography. Additionally, the confirmation tests reported here were performed by physicians well versed in the post Essure confirmation test guidelines and interpretation. We assume that higher-pressure "infertility-style" hysterosalpinograms may be associated with higher degrees of pain. An additional limitation is that while all patients verified that they had premedicated themselves with a nonsteroidal anti-inflammatory agent, we did not break down medication dosage or ingestion interval-hysterosalpinography performance. However, as previously stated, this study was not intended, powered, nor designed to evaluate the "best" medication regimen before hysterosalpinography.

Obstetricians and gynecologists are projected to see an increase in patient volume/load due to provisions within the Patient Protection and Affordable Care Act. No-cost contraception under third party insurance will undoubtedly increase the demand for patient education and services regarding sterilization. Our study may provide reassurance; in that post hysteroscopic sterilization confirmation testing is well tolerated. Given that no prior literature has evaluated the post Essure confirmation test from a patient perspective, we feel this information will aid the physician in the informed consent process for reviewing permanent birth control options with our patients.

\section{Disclosure}

HOC serves as a medical consultant for Conceptus Inc. Conceptus Inc. provided financial support for publication costs after manuscript acceptance by peer review. This study was conducted as an independent nonfunded investigation. GV has no disclosures relevant to this work.

\section{References}

1. Zite N, Borrero S. Female sterilisation in the United States. Eur J Contracept Reprod Health Care. 2011;16(5):336-340.

2. Chapa HO, Antonetti AG, Sandate J. Office versus hospital based Essure $^{\circledR}$ procedure: pareto principle in action. J Gynecol Surg. 2012; 28(1):16-19.

3. Connor VF. Essure: a review six years later. J Minim Invasive Gynecol. 2009; 16(3):282-290.

4. Levie M, Weiss G, Kaiser B, Daif J, Chudnoff SG. Analysis of pain and satisfaction with office-based hysteroscopic sterilization. Fertil Steril. 2010;94(4):1189-1194.

5. Duffy S, Marsh F, Rogerson L, et al. Female sterilisation: a cohort controlled comparative study of ESSURE versus laparoscopic sterilisation. BJOG. 2005;112(11):1522-1528.

6. Nichols M, Carter JF, Fylstra DL, Childers M. A comparative study of hysteroscopic sterilization performed in-office versus a hospital operating room. J Minim Invasive Gynecol. 2006;13(5):447-450.

7. Karasahin E, Alanbay I, Keskin U, Gezginc K, Baser I. Lidocaine. $10 \%$ spray reduces pain during hysterosalpingography: a randomized controlled trial. J Obstet Gynaecol Res. 2009;35(2):354-358.

8. Zhu YY, Mao YZ, Wu WL. Comparison of warm and cold contrast media for hysterosalpingography: a prospective, randomized study. Fertil Steril. 2012;97(6):1405-1409.

9. Chauhan MB, Lakra P, Jyotsna D, Nanda S, Malhotra V. Pain relief during hysterosalpingography: role of intracervical block. Arch Gynecol Obstet. 2013;287(1):155-159.

10. Chapa HO, Venegas G. Preprocedure patient preferences and attitudes toward permanent contraceptive options. Patient Prefer Adherence. 2012;6:331-336.

11. Hamill-Ruth RJ, Marohn ML. Evaluation of pain in the critically ill patient. Crit Care Clin. 1999;15(1):35-54. 
12. Levie MD, Chudnoff S. Prospective analysis of office-based hysteroscopic sterilization. J Minim Invasive Gynecol. 2006;13(2):98-101.

13. Arjona JE, Miño M, Cordón J, Povedano B, Pelegrin B, Castelo-Branco C. Satisfaction and tolerance with office hysteroscopic tubal sterilization. Fertil Steril. 2008;90(4):1182-1186.

14. Castaño PM, Adekunle L. Transcervical sterilization. Semin Reprod Med. 2010;28(2):103-109.

15. Cleary TP, Tepper NK, Cwiak C, et al. Pregnancies after hysteroscopic sterilization: a systematic review. Contraception. 2012; pii:S0010-7824(12)00733-0.

16. Povedano B, Arjona JE, Velasco E, Monserrat JA, Lorente J, CasteloBranco C. Complications of hysteroscopic Essure ${ }^{\circledR}$ sterilisation: report on 4306 procedures performed in a single centre. BJOG. 2012; 119(7):795-799.
17. Howard DL, Wall J, Strickland JL. What are the factors predictive of hysterosalpingogram compliance after female sterilization by the Essure procedure in a publicly insured population? Matern Child Health J. December 5, 2012. [Epub ahead of print.]

18. The Essure Confirmation Test. Available from: http://essuremd.com/ Home/MyEssureMD/RadiologyResourceCenter/tabid/759/Default. aspx. Accessed February 17, 2013.

19. Updike GM, Manolitsas TP, Cohn DE, et al. Pre-emptive analgesia in gynecologic surgical procedures: preoperative wound infiltration with ropivacaine in patients who undergo laparotomy through a midline vertical incision. Am J Obstet Gynecol. 2003;188(4):901-905.

20. Tripp DA, Stanish WD, Reardon G, Coady C, Sullivan MJL. Comparing postoperative pain experiences of the adolescent and adult athlete after anterior cruciate ligament surgery. J Athl Train. 2003;38(2):154-157.

\section{Publish your work in this journal}

Patient Preference and Adherence is an international, peer-reviewed, open access journal focusing on the growing importance of patient preference and adherence throughout the therapeutic continuum. Patient satisfaction, acceptability, quality of life, compliance, persistence and their role in developing new therapeutic modalities and compounds to optimize clinical outcomes for existing disease states are major areas of interest. This journal has been accepted for indexing on PubMed Central. The manuscript management system is completely online and includes a very quick and fair peer-review system. Visit http://www.dovepress.com/ testimonials.php to read real quotes from published authors. 